\title{
MEASUREMENT AND ANALYSIS OF FIELD EMISSION ELECTRONS IN THE LCLS GUN*
}

\author{
D.H. Dowell\#, E. Jongewaard, C. Limborg-Deprey, J.F. Schmerge and A. Vlieks \\ SLAC, Menlo Park, CA 24059, U.S.A.
}

\begin{abstract}
The field emission was measured during the highpower testing of the LCLS photocathode RF gun. A careful study and analysis of the field emission electrons, or dark current is important in assessing the gun's internal surface quality in actual operation, especially those surfaces with high fields. The first indication of a good $\mathrm{RF}$ gun design and fabrication is short processing time to the required fields and low electron emission at highfields. The charge per 2 microsecond long RF pulse (the dark charge) was measured as a function of the peak cathode field for the 1.6 cell, $2.856 \mathrm{GHz}$ LCLS RF gun. Faraday cup data was taken for cathode peak RF fields up to $120 \mathrm{MV} / \mathrm{m}$ producing a maximum of $0.6 \mathrm{nC} / \mathrm{RF}$ pulse for a diamond-turned polycrystalline copper cathode installed in the gun. Digitized images of the dark charge were taken using a 100 micron thick YAG crystal for a range of solenoid fields to determine the location and angular distribution of the field emitters. The FN plots and emitter image analysis will be described in this paper.
\end{abstract}

\section{INTRODUCTION}

The goal of this paper is to demonstrate the application of a relativity simple linear optical model for the RF gun and focusing solenoid to the dark charge images observed during high-power RF processing of the LCLS gun. The RF details of these tests are given in another contribution to these proceedings[1].

The dark charge images where measured using a 100 micron thick YAG crystal normal to the beam axis followed by a 45 degree mirror. The mirror reflects the scintillation light through a vacuum window to be viewed by a digital camera. A photograph of this arrangement is shown in Figure 1.

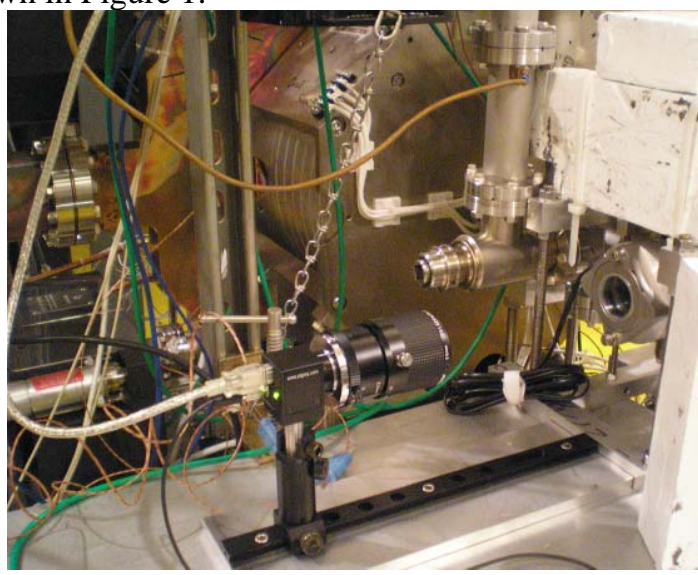

Figure 1: Photograph of camera and vacuum port used to view the YAG screen. The gun solenoid is left of the YAG vacuum cross and behind the camera.
A typical image of the dark current is shown in Figure 2. Each of the arcs is produced by electrons emitted from a point emitter on the cathode with a large energy spread due to the time varying RF field.

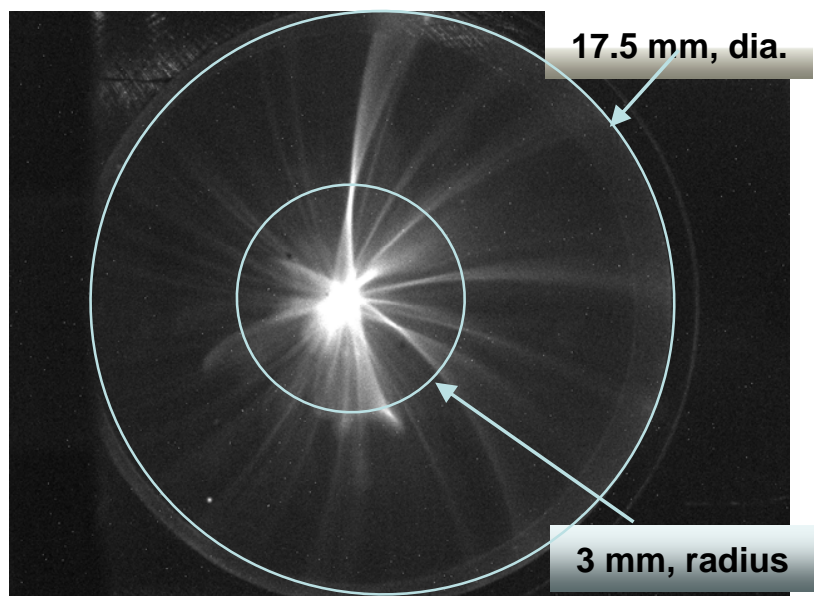

Figure 2: Image of the dark charge on the YAG screen with the solenoid field adjusted to collect the dark charge.

\section{FIRST-ORDER MODEL OF THE GUN\&SOLENOID}

In the absence of space charge the electron gun transverse dynamics can be approximated by drift lengths and thin lenses. Using the numerical calculation of the beam trajectories in realistic fields shown in Figure 3, we observe the gun behaves like a simple defocusing lens as indicated by the divergent trajectories.

Therefore we consider a 'toy' model of the gun and solenoid consisting of a thin defocusing lens for the gun followed by a thick solenoid lens. as shown in Figure 4.

The gun lens focal length is given by [2]

$$
f_{\text {gun }}=-\frac{m c^{2}}{E_{0} \sin \phi_{\text {exit }}}
$$

where $\mathrm{mc}^{2}$ is the electron rest mass, $\mathrm{E}_{0}$ is the RF electric field and $\phi_{\text {exit }}$ is the synchronous electron phase at the exit of the gun. The gun transport is then represented by a drift, $L_{1}$, from the cathode to the thin lens and another drift, $L_{2}$, to the entrance of the solenoid.

\footnotetext{
* Work supported by US DOE contract DE-AC02-76SF00515.

\# dowell@SLAC.Stanford.edu
} 
$R_{\text {gun }}=\left(\begin{array}{cccc}1 & L_{2} & 0 & 0 \\ 0 & 1 & 0 & 0 \\ 0 & 0 & 1 & L_{2} \\ 0 & 0 & 0 & 1\end{array}\right)\left(\begin{array}{cccc}1 & 0 & 0 & 0 \\ -\frac{1}{f_{\text {gun }}} & 1 & 0 & 0 \\ 0 & 0 & 1 & 0 \\ 0 & 0 & -\frac{1}{f_{\text {gun }}} & 1\end{array}\right)\left(\begin{array}{cccc}1 & L_{1} & 0 & 0 \\ 0 & 1 & 0 & 0 \\ 0 & 0 & 1 & L_{1} \\ 0 & 0 & 0 & 1\end{array}\right)$

The transformation matrix for a magnetic solenoid is given by [ref]

$$
R_{\text {solenoid }}=\left(\begin{array}{cccc}
C^{2} & \frac{S C}{K} & S C & \frac{S^{2}}{K} \\
-K S C & C^{2} & -K S^{2} & S C \\
-S C & \frac{-S^{2}}{K} & C^{2} & \frac{S C}{K} \\
K S^{2} & -S C & -K S C & C^{2}
\end{array}\right)
$$

Where $\mathrm{K}$ is the solenoid wave number, and $\mathrm{C}$ and $\mathrm{S}$ are the cosine and sine of the beam's rotation angle, $\mathrm{KL}_{\text {solenoid }}$ in the solenoid field which is dependent upon the bema momentum,

$$
\begin{aligned}
& K\left(B_{\text {solenoid }}, p_{\text {beam }}\right)=\frac{B_{\text {solenoid }}(k G)}{2 \cdot 0.033356 p_{\text {beam }}(M e V / c)}\left(m^{-1}\right) \\
& C=\cos \left(K L_{\text {solenoid }}\right) \text { and } S=\sin \left(K L_{\text {solenoid }}\right) .
\end{aligned}
$$

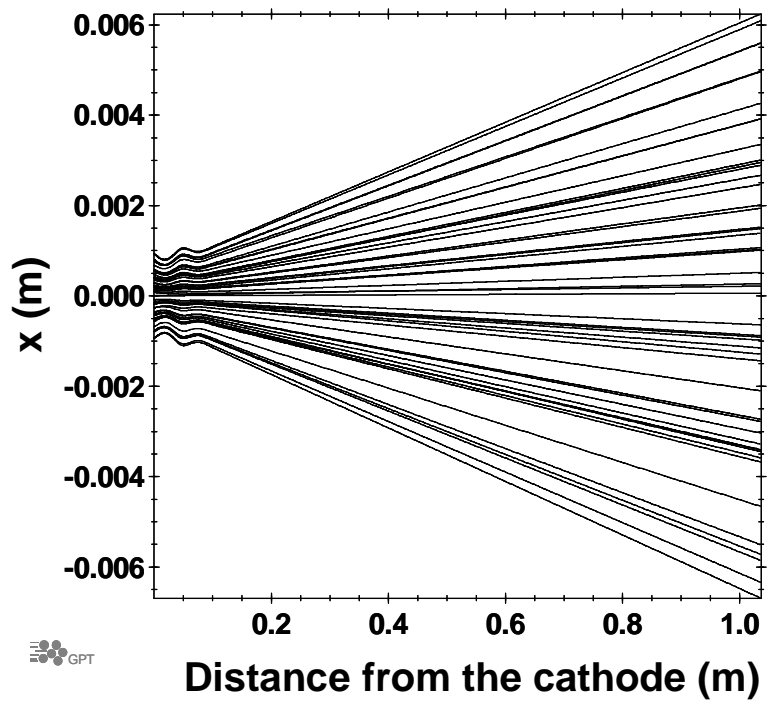

Figure 3: Numerical calculation [3] of beam trajectories in the gun using the exact RF fields.

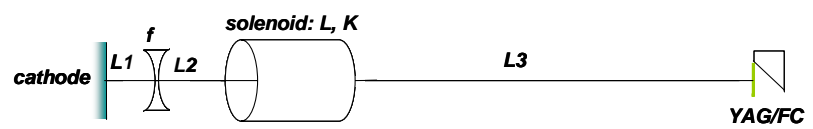

Figure 4: The 'toy' model of the RF gun and solenoid used for analyzing the dark charge.

Finally the complete transformation from the cathode to the YAG screen is then given by,

$$
R_{Y A G}=\left(\begin{array}{cccc}
1 & L_{3} & 0 & 0 \\
0 & 1 & 0 & 0 \\
0 & 0 & 1 & L_{3} \\
0 & 0 & 0 & 1
\end{array}\right) \times R_{\text {solenoid }} R_{\text {gun }}
$$

The matrix elements of the overall transformation are plotted in Figure 5.

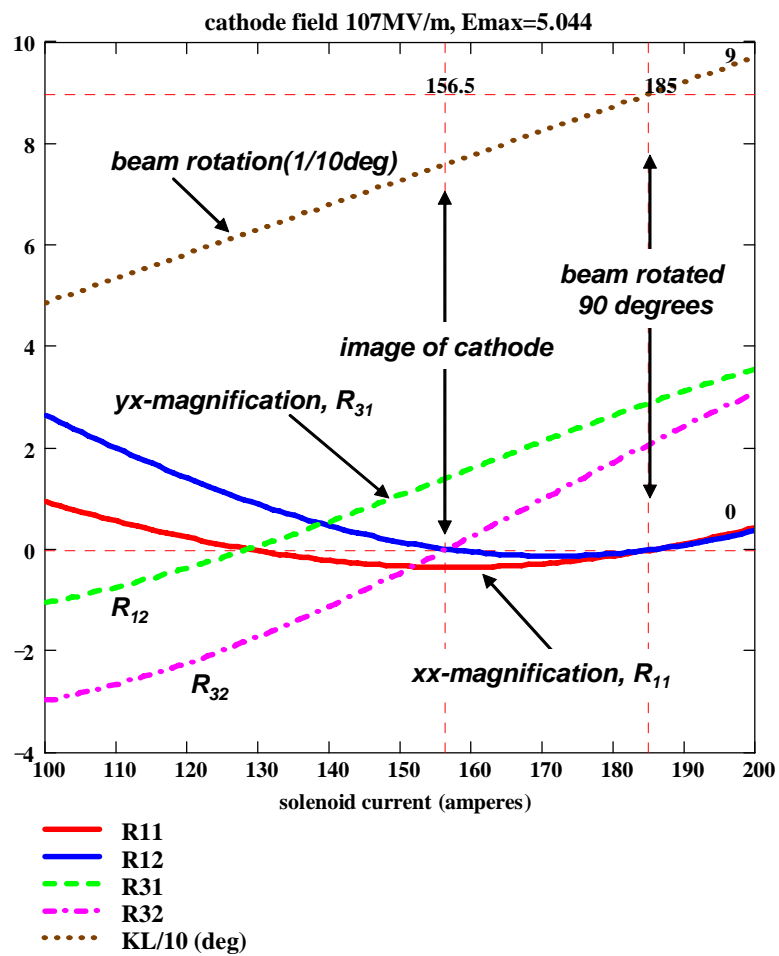

Figure 5: Plots of the matrix elements of the gun + solenoid transformation.

The matrix elements and the beam rotation angle for this transformation are plotted in Figure 5 as a function of the solenoid current for a gun electric field of $107 \mathrm{MV} / \mathrm{m}$. For this case, the cathode surface is imaged on the YAG screen (when $\mathrm{R}_{12}$ is zero) at 156.5 amperes where the beam rotation is approximately 75 degrees.

\section{COMPARISON WITH EXPERIMENTAL RESULTS}

Figures 6 and 7 show measured YAG images of the dark charge for solenoid currents of $x, y$, and $z$. The calculation of the $x-y$ position of the beam position for large energy spread is plotted below each video image. The calculations assume the emitters on the cathode surface are from points distributed across the surface with a small initial angle. The initial conditions are given for each figure.

This simple 'toy' model replicates the major features of the beam images. It explains the bright 'spot' in the center by the overlap of the large energy spread induced 
arcs when the solenoid is not imaging the cathode. The end of each 'arc' is where the electron energy is the highest. The location of the emitters on the cathode surface can be seen in Figure 7 when the solenoid has been adjusted to image the cathode.
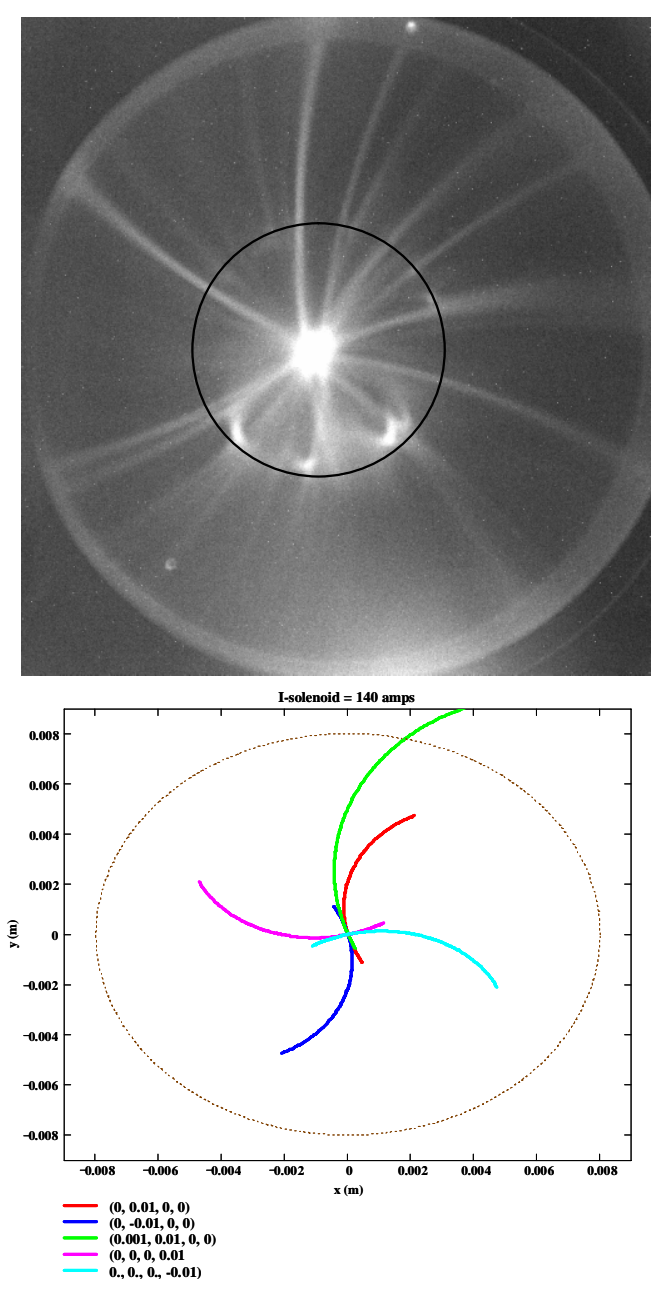

Figure 6: Top: Image of the dark charge with the solenoid set to 140 amperes. Bottom: Electron trajectories computed using the linear model.
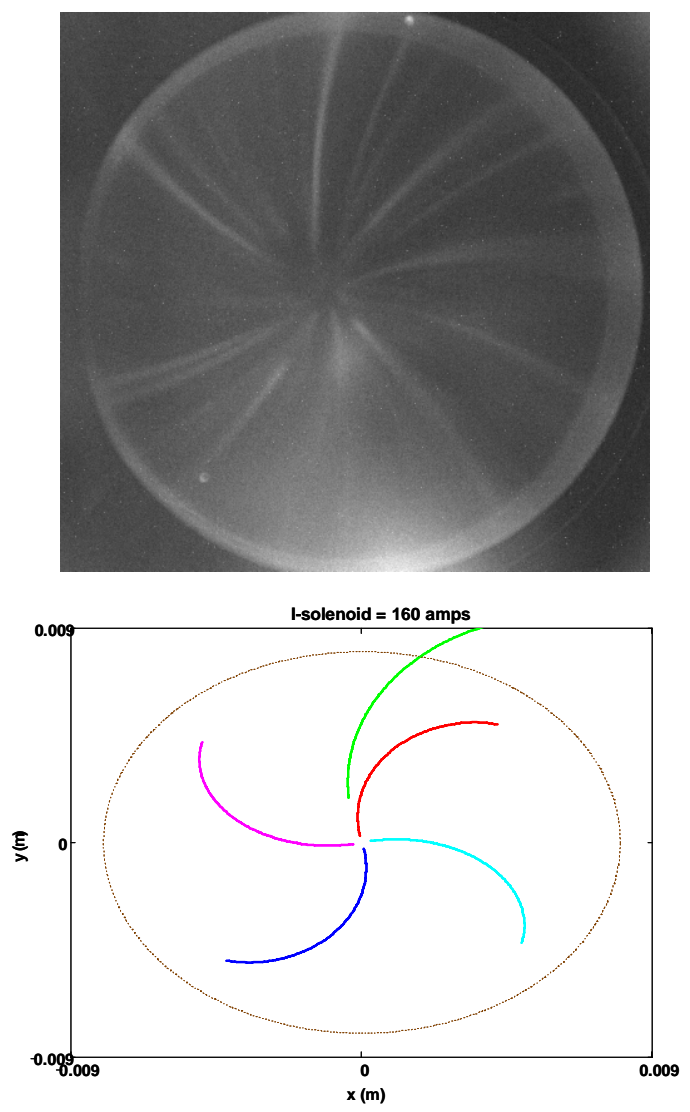

Figure 7: Top: Image on YAG screen with solenoid at 160 amperes. Bottom: Image as computed using the linear model.

\section{CONCLUSIONS}

A linear model has been used to analyze the images of the dark charge from the LCLS gun. This model provides a good representation of the observed results and provides useful physical insight of the field emission sources.

\section{REFERENCES}

[1] D. H. Dowell, E. Jongewaard, C. Limborg-Deprey, J. Schmerge, Z. Li, L. Xiao, J. Wang, J. Lewandowski, A. Vlieks, "Results of the SLAC LCLS Gun High-Power RF Tests", These conference proceedings.

[2] K.-J. Kim, NIM 1989.

[3] General Particle Tracer, GPT, Pulsar Physics, www.pulsar.nl. 